\title{
Estimation of the occurrence of drought in Poland by 2060 based on the HTC index and probability distributions
}

\author{
Ewa Diakowska ${ }^{1, *}$, Paulina Stanek $^{1,}$, Stawomir Iwański ${ }^{1}$ and Edward Gąsiorek ${ }^{1}$ \\ ${ }^{1}$ Wrocław University of Environmental and Life Sciences, Department of Mathematics, Grunwaldzka 53, 50-357 Wrocław, Poland
}

\begin{abstract}
This article attempts to assess the future occurrence of a drought using the Sieljaninova hydrothermal index (HTC). Air temperature and total precipitation were simulated for current conditions (2000) and forecast for eight locations of meteorological stations in Poland according to the HAD and GFDL scenarios for RCP 45 and 60 for 2040 and 2060.
\end{abstract}

\section{Introduction}

The study of climate variability is a topic of interest for many scientists in many diverse fields, including, among others, hydrologists, meteorologists, farmers, and foresters. All would like to determine as precisely as possible what climatic conditions will prevail in a given area in the future $[1-10]$. Greater computing power allows us to analyze increasingly complex models while showing that there are more factors affecting the environment. Therefore, the problem remains unresolved.

In accordance with various scenarios of climate change for Central Europe, the temperature increase will be accompanied by a very small increase in annual rainfall which will be redistributed over the year - an increase in the winter will be accompanied by a decline in the summer [11 - 14]. In this situation with poor retention possibilities and a simultaneous increase in evaporation, it should be expected that the amount of water that is useful for plants will be reduced during the growing season, with the possible depletion of postwinter stocks. As the authors of the works $[15,16]$ indicate, one must not neglect the effect of the growing variance of precipitation and temperatures, which means that extreme situations which are unfavorable in plant production will occur more frequently.

One elements to observe is the assessment of the amount of water present in a given area, especially in extreme values, i.e. floods and droughts. Different types of indicators are used to assess the severity of water shortages. One of these is the Sjeljaninowa hydrothermal indicator (HTC) [17, 18]. The pattern, which has been tested by the Russian researcher, assesses drought according to the following formula:

$$
\mathrm{HTC}=\frac{10 \sum_{i=1}^{n_{i}} p_{i}}{\sum_{i=1}^{n} \mathrm{t}_{i}}
$$

where:

$n$ - length of the period considered in days,
$P_{\mathrm{i}}$ - rainfall on the $i$-th day [mm],

$t_{i}$ - average daily temperature on the $i$-th day $\left[{ }^{\circ} \mathrm{C}\right]$.

The indicator is properly interpreted when $t_{i}>10^{\circ} \mathrm{C}$, i.e. for average daily temperatures greater than $10^{\circ} \mathrm{C}$ according to the following scale: extremely dry $(\mathrm{HTC} \leq$ $0.4)$, very dry $(0.4<\mathrm{HTC} \leq 0.7)$, dry $(0.7<\mathrm{HTC} \leq 1.0)$, quite dry $(1.0<\mathrm{HTC} \leq 1.3)$ and optimal $(1.3<\mathrm{HTC} \leq$ 1.6).

Long-term water shortages cause significant losses particularly in agricultural areas. Therefore, drought monitoring is carried out primarily during the growing season (from April 1 to September 30). The advantage of this indicator over others is the fact that only readily available information is needed to determine it, i.e. the amount of rainfall and average daily temperature [1920]. Strings of any length can be considered, however, those most frequently analyzed are 15-day and 30-day observation strings.

\section{Materials and methods}

The idea of simulating spatial meteorological data using a spatial generator is widely described in the literature [21 - 24]. The SWGEN model generates two-stage precipitation. First, it uses the a first-order Markov chain to determine the state of the day (whether precipitation occurred or did not occur), then the precipitation is obtained by correlating two-parameter gamma distributions. Different values for solar radiation (SR), maximum temperature (Tmax) and minimum temperature (Tmin) are generated by means of multidimensional time series AR(1). According to the SWGEN model, the required characteristics were determined for each station (mean value, standard deviation, daily sum of rainfall, transition probabilities, correlations) for the following variables: solar radiation (SR), minimum and maximum temperature (Tmin and Tmax). 
The data simulation was applied to eight sites located in central Poland (Fig. 1). Daily data of maximum and minimum air temperature, and total precipitation from a 30-year data series (1981-2010) of the meteorological network within the study area were obtained from the Institute of Meteorology and Water Management National Research Institute. Evaluation was made of the hydrothermal conditions for current climate conditions (simulations for 2000) and of conditions for the time horizon of 2040 and 2060.

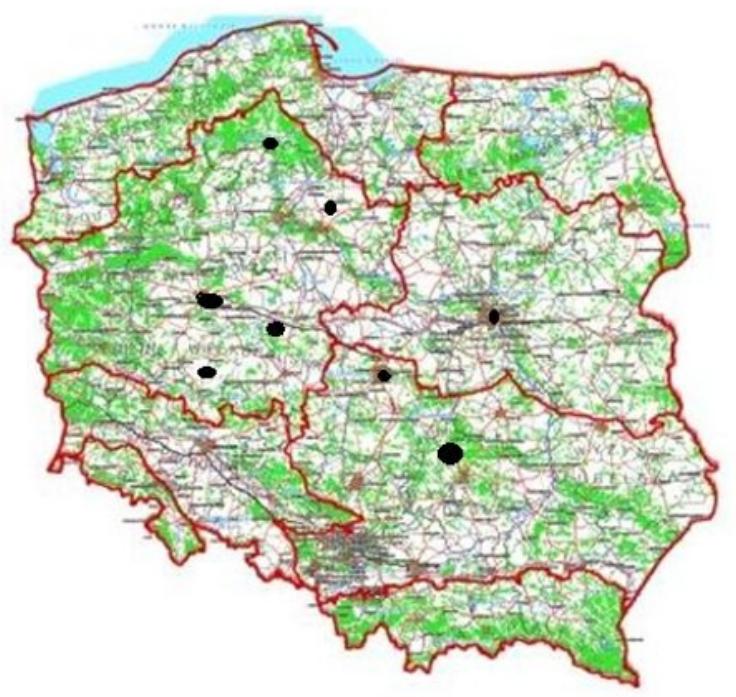

Fig. 1. Location of measurement stations in the territory of Poland.

\section{Results}

For the time horizon to 2040, the probability of the occurrence of individual drought conditions for selected cities was calculated. Each month was regarded as a change to which the schedule was adjusted. Gamma distribution was chosen because it best suited the existing data. With the help of $\mathrm{R}$ software (An $\mathrm{R}$ Package for Fitting Distribution), the parameters of this distribution were estimated for each month and then the probabilities were determined. The results are showned in Tables $1-8$. The same calculations were made for the time horizon 2060 (Tab. 9 - 16).

Table 1. Extremely dry conditions (HTC $\leq 0.4)$, model GFDL, RCP 45, 2040.

\begin{tabular}{|c|c|c|c|c|c|}
\hline City & IV & V & VI & VII & VIII \\
\hline Chojnice & $1.6 \%$ & $3.0 \%$ & $2.9 \%$ & $4.5 \%$ & $1.5 \%$ \\
\hline Kalisz & $5.5 \%$ & $4.1 \%$ & $3.8 \%$ & $8.4 \%$ & $3.6 \%$ \\
\hline Kielce & $1.0 \%$ & $0.9 \%$ & $0.6 \%$ & $1.6 \%$ & $1.6 \%$ \\
\hline Leszno & $4.5 \%$ & $4.7 \%$ & $2.9 \%$ & $5.4 \%$ & $4.7 \%$ \\
\hline Łódź & $3.8 \%$ & $2.9 \%$ & $2.1 \%$ & $12.6 \%$ & $1.7 \%$ \\
\hline Poznań & $5.9 \%$ & $4.4 \%$ & $2.2 \%$ & $8.6 \%$ & $3.7 \%$ \\
\hline Toruń & $4.3 \%$ & $5.1 \%$ & $3.3 \%$ & $6.1 \%$ & $1.9 \%$ \\
\hline Warszawa & $2.9 \%$ & $3.4 \%$ & $4.6 \%$ & $9.4 \%$ & $3.1 \%$ \\
\hline
\end{tabular}

Table 2. Extremely dry or very dry conditions $(\mathrm{HTC} \leq 0.7)$, model GFDL, RCP 45, 2040.

\begin{tabular}{|c|c|c|c|c|c|}
\hline City & IV & V & VI & VII & VIII \\
\hline Chojnice & $9.1 \%$ & $19.3 \%$ & $18.1 \%$ & $20.8 \%$ & $10.0 \%$ \\
\hline Kalisz & $22.5 \%$ & $26.3 \%$ & $20.3 \%$ & $35.8 \%$ & $19.1 \%$ \\
\hline Kielce & $6.5 \%$ & $10.0 \%$ & $6.0 \%$ & $12.4 \%$ & $10.5 \%$ \\
\hline Leszno & $19.0 \%$ & $26.0 \%$ & $16.7 \%$ & $24.3 \%$ & $23.0 \%$ \\
\hline Łódź & $15.9 \%$ & $20.7 \%$ & $13.3 \%$ & $42.6 \%$ & $12.2 \%$ \\
\hline Poznań & $23.0 \%$ & $23.2 \%$ & $14.3 \%$ & $34.4 \%$ & $18.2 \%$ \\
\hline Toruń & $20.5 \%$ & $29.7 \%$ & $16.5 \%$ & $27.3 \%$ & $11.2 \%$ \\
\hline Warszawa & $16.0 \%$ & $20.1 \%$ & $21.0 \%$ & $35.9 \%$ & $16.6 \%$ \\
\hline
\end{tabular}

Table 3. Extremely dry conditions ( $\mathrm{HTC} \leq 0.4)$, HAD model, RCP 45, 2040.

\begin{tabular}{|c|c|c|c|c|c|}
\hline City & IV & V & VI & VII & VIII \\
\hline Chojnice & $3.2 \%$ & $2.3 \%$ & $3.3 \%$ & $3.5 \%$ & $0.7 \%$ \\
\hline Kalisz & $5.7 \%$ & $5.9 \%$ & $3.0 \%$ & $9.7 \%$ & $3.8 \%$ \\
\hline Kielce & $2.2 \%$ & $1.0 \%$ & $0.5 \%$ & $1.6 \%$ & $2.2 \%$ \\
\hline Leszno & $2.2 \%$ & $4.1 \%$ & $2.7 \%$ & $6.3 \%$ & $3.5 \%$ \\
\hline Łódź & $4.7 \%$ & $3.2 \%$ & $1.7 \%$ & $11.8 \%$ & $2.4 \%$ \\
\hline Poznań & $6.0 \%$ & $4.0 \%$ & $2.8 \%$ & $6.7 \%$ & $3.2 \%$ \\
\hline Toruń & $6.8 \%$ & $4.6 \%$ & $2.7 \%$ & $4.3 \%$ & $1.5 \%$ \\
\hline Warszawa & $6.0 \%$ & $2.8 \%$ & $3.8 \%$ & $8.1 \%$ & $2.6 \%$ \\
\hline
\end{tabular}

Table 4. Extremely dry or very dry conditions (HTC $\leq 0.7)$, HAD model, RCP 45, 2040.

\begin{tabular}{|c|c|c|c|c|c|}
\hline City & IV & V & VI & VII & VIII \\
\hline Chojnice & $15.2 \%$ & $17.1 \%$ & $16.7 \%$ & $16.9 \%$ & $7.2 \%$ \\
\hline Kalisz & $24.1 \%$ & $26.7 \%$ & $16.7 \%$ & $34.4 \%$ & $19.2 \%$ \\
\hline Kielce & $11.2 \%$ & $9.5 \%$ & $4.7 \%$ & $10.9 \%$ & $12.9 \%$ \\
\hline Leszno & $12.9 \%$ & $23.6 \%$ & $14.8 \%$ & $24.2 \%$ & $20.6 \%$ \\
\hline Łódź & $19.1 \%$ & $20.9 \%$ & $11.2 \%$ & $37.7 \%$ & $15.1 \%$ \\
\hline Poznań & $25.3 \%$ & $21.1 \%$ & $14.2 \%$ & $29.9 \%$ & $17.7 \%$ \\
\hline Toruń & $27.1 \%$ & $26.4 \%$ & $13.9 \%$ & $21.5 \%$ & $10.4 \%$ \\
\hline Warszawa & $24.0 \%$ & $17.7 \%$ & $17.2 \%$ & $30.1 \%$ & $16.0 \%$ \\
\hline
\end{tabular}

Table 5. Extremely dry conditions (HTC $\leq 0.4$ ), model GFDL, RCP 60, 2040.

\begin{tabular}{|c|c|c|c|c|c|}
\hline City & IV & V & VI & VII & VIII \\
\hline Chojnice & $3.7 \%$ & $1.8 \%$ & $2.1 \%$ & $3.7 \%$ & $2.2 \%$ \\
\hline Kalisz & $5.9 \%$ & $3.7 \%$ & $2.4 \%$ & $6.1 \%$ & $5.3 \%$ \\
\hline Kielce & $2.6 \%$ & $1.3 \%$ & $0.3 \%$ & $1.5 \%$ & $1.8 \%$ \\
\hline Leszno & $6.9 \%$ & $4.5 \%$ & $1.9 \%$ & $2.8 \%$ & $2.8 \%$ \\
\hline Łódź & $5.0 \%$ & $3.1 \%$ & $1.8 \%$ & $10.4 \%$ & $2.1 \%$ \\
\hline Poznań & $9.9 \%$ & $3.3 \%$ & $1.5 \%$ & $7.5 \%$ & $3.8 \%$ \\
\hline Toruń & $1.0 \%$ & $1.4 \%$ & $0.7 \%$ & $1.5 \%$ & $2.4 \%$ \\
\hline Warszawa & $6.1 \%$ & $2.3 \%$ & $4.2 \%$ & $7.0 \%$ & $3.8 \%$ \\
\hline
\end{tabular}


Table 6. Extremely dry or very dry conditions $(\mathrm{HTC} \leq 0.7)$, model GFDL, RCP 60, 2040.

\begin{tabular}{|c|c|c|c|c|c|}
\hline City & IV & V & VI & VII & VIII \\
\hline Chojnice & $18.2 \%$ & $13.2 \%$ & $13.7 \%$ & $17.0 \%$ & $13.9 \%$ \\
\hline Kalisz & $24.6 \%$ & $21.1 \%$ & $14.2 \%$ & $29.0 \%$ & $26.2 \%$ \\
\hline Kielce & $12.0 \%$ & $10.1 \%$ & $3.6 \%$ & $10.4 \%$ & $13.6 \%$ \\
\hline Leszno & $24.0 \%$ & $21.9 \%$ & $11.9 \%$ & $16.6 \%$ & $16.6 \%$ \\
\hline Łódź & $18.8 \%$ & $17.9 \%$ & $10.8 \%$ & $36.3 \%$ & $16.0 \%$ \\
\hline Poznań & $31.2 \%$ & $17.3 \%$ & $10.4 \%$ & $28.9 \%$ & $21.9 \%$ \\
\hline Toruń & $6.9 \%$ & $11.5 \%$ & $6.4 \%$ & $11.8 \%$ & $13.2 \%$ \\
\hline Warszawa & $23.6 \%$ & $15.2 \%$ & $18.2 \%$ & $28.9 \%$ & $20.8 \%$ \\
\hline
\end{tabular}

Table 7. Extremely dry conditions (HTC $\leq 0.4)$, HAD model, RCP 60, 2040.

\begin{tabular}{|c|c|c|c|c|c|}
\hline City & IV & V & VI & VII & VIII \\
\hline Chojnice & $13.3 \%$ & $11.6 \%$ & $6.7 \%$ & $6.8 \%$ & $7.5 \%$ \\
\hline Kalisz & $17.5 \%$ & $7.8 \%$ & $5.1 \%$ & $11.3 \%$ & $14.7 \%$ \\
\hline Kielce & $9.5 \%$ & $5.2 \%$ & $2.1 \%$ & $2.5 \%$ & $9.5 \%$ \\
\hline Leszno & $13.7 \%$ & $7.7 \%$ & $3.7 \%$ & $6.1 \%$ & $19.1 \%$ \\
\hline Lódź & $15.0 \%$ & $11.0 \%$ & $4.6 \%$ & $15.1 \%$ & $8.7 \%$ \\
\hline Poznań & $21.3 \%$ & $7.6 \%$ & $3.2 \%$ & $10.9 \%$ & $15.3 \%$ \\
\hline Toruń & $24.1 \%$ & $15.9 \%$ & $5.0 \%$ & $8.9 \%$ & $9.9 \%$ \\
\hline Warszawa & $22.8 \%$ & $10.7 \%$ & $8.0 \%$ & $8.0 \%$ & $11.1 \%$ \\
\hline
\end{tabular}

Table 8. Extremely dry or very dry conditions $(\mathrm{HTC} \leq 0.7)$, HAD model, RCP 60, 2040.

\begin{tabular}{|c|c|c|c|c|c|}
\hline City & IV & V & VI & VII & VIII \\
\hline Chojnice & $13.3 \%$ & $11.6 \%$ & $6.7 \%$ & $6.8 \%$ & $7.5 \%$ \\
\hline Kalisz & $17.5 \%$ & $7.8 \%$ & $5.1 \%$ & $11.3 \%$ & $14.7 \%$ \\
\hline Kielce & $9.5 \%$ & $5.2 \%$ & $2.1 \%$ & $2.5 \%$ & $9.5 \%$ \\
\hline Leszno & $13.7 \%$ & $7.7 \%$ & $3.7 \%$ & $6.1 \%$ & $19.1 \%$ \\
\hline Łódź & $15.0 \%$ & $11.0 \%$ & $4.6 \%$ & $15.1 \%$ & $8.7 \%$ \\
\hline Poznań & $21.3 \%$ & $7.6 \%$ & $3.2 \%$ & $10.9 \%$ & $15.3 \%$ \\
\hline Toruń & $24.1 \%$ & $15.9 \%$ & $5.0 \%$ & $8.9 \%$ & $9.9 \%$ \\
\hline Warszawa & $22.8 \%$ & $10.7 \%$ & $8.0 \%$ & $8.0 \%$ & $11.1 \%$ \\
\hline
\end{tabular}

Table 9. Extremely dry conditions (HTC $\leq 0.4)$, model GFDL, RCP 45, 2060.

\begin{tabular}{|c|c|c|c|c|c|}
\hline City & IV & V & VI & VII & VIII \\
\hline Chojnice & $2.3 \%$ & $3.5 \%$ & $2.9 \%$ & $4.2 \%$ & $2.2 \%$ \\
\hline Kalisz & $5.0 \%$ & $4.1 \%$ & $3.5 \%$ & $10.3 \%$ & $4.9 \%$ \\
\hline Kielce & $1.6 \%$ & $1.7 \%$ & $0.7 \%$ & $2.0 \%$ & $3.0 \%$ \\
\hline Leszno & $4.2 \%$ & $6.7 \%$ & $2,1 \%$ & $4,7 \%$ & $4.8 \%$ \\
\hline Łódź & $4.8 \%$ & $4.4 \%$ & $2.0 \%$ & $12.1 \%$ & $2.4 \%$ \\
\hline Poznań & $6.8 \%$ & $4.3 \%$ & $1.5 \%$ & $8.7 \%$ & $5.1 \%$ \\
\hline Toruń & $6.3 \%$ & $4.4 \%$ & $2.2 \%$ & $9.7 \%$ & $2.0 \%$ \\
\hline Warszawa & $4.1 \%$ & $2.9 \%$ & $4.1 \%$ & $9.6 \%$ & $3.7 \%$ \\
\hline
\end{tabular}

Table 10. Extremely dry or very dry conditions (HTC $\leq 0.7)$, model GFDL, RCP 45, 2060.

\begin{tabular}{|c|c|c|c|c|c|}
\hline City & IV & V & VI & VII & VIII \\
\hline Chojnice & $11.5 \%$ & $19.9 \%$ & $17.4 \%$ & $20.7 \%$ & $12.6 \%$ \\
\hline Kalisz & $22.5 \%$ & $25.8 \%$ & $19.4 \%$ & $38.9 \%$ & $22.0 \%$ \\
\hline Kielce & $9.0 \%$ & $12.9 \%$ & $6.5 \%$ & $13.3 \%$ & $15.3 \%$ \\
\hline Leszno & $19.3 \%$ & $28.5 \%$ & $14.7 \%$ & $22.4 \%$ & $23.2 \%$ \\
\hline Łódź & $23.2 \%$ & $22.9 \%$ & $13.2 \%$ & $42.6 \%$ & $15.4 \%$ \\
\hline Poznań & $24.9 \%$ & $23.4 \%$ & $11.6 \%$ & $35.2 \%$ & $21.7 \%$ \\
\hline Toruń & $24.3 \%$ & $27.5 \%$ & $13.5 \%$ & $32.9 \%$ & $12.2 \%$ \\
\hline Warszawa & $18.7 \%$ & $19.4 \%$ & $19.6 \%$ & $36.1 \%$ & $18.2 \%$ \\
\hline
\end{tabular}

Table 11. Extremely dry conditions (HTC $\leq 0.4)$, HAD model, RCP 45, 2060.

\begin{tabular}{|c|c|c|c|c|c|}
\hline City & IV & V & VI & VII & VIII \\
\hline Chojnice & $4.2 \%$ & $3.0 \%$ & $3.3 \%$ & $4.6 \%$ & $1.2 \%$ \\
\hline Kalisz & $5.8 \%$ & $5.7 \%$ & $3.1 \%$ & $8.4 \%$ & $3.2 \%$ \\
\hline Kielce & $2.5 \%$ & $1.9 \%$ & $0.5 \%$ & $1.7 \%$ & $1.8 \%$ \\
\hline Leszno & $5.8 \%$ & $4.7 \%$ & $2.7 \%$ & $3.9 \%$ & $3.5 \%$ \\
\hline Łódź & $4.5 \%$ & $4.2 \%$ & $1.7 \%$ & $11.3 \%$ & $1.3 \%$ \\
\hline Poznań & $8.7 \%$ & $4.1 \%$ & $2.4 \%$ & $6.6 \%$ & $6.6 \%$ \\
\hline Toruń & $8.0 \%$ & $4.9 \%$ & $2.0 \%$ & $6.8 \%$ & $1.5 \%$ \\
\hline Warszawa & $6.4 \%$ & $2.8 \%$ & $3.7 \%$ & $8.4 \%$ & $2.0 \%$ \\
\hline
\end{tabular}

Table 12. Extremely dry or very dry conditions $(\mathrm{HTC} \leq 0.7)$, HAD model, RCP 45, 2060.

\begin{tabular}{|c|c|c|c|c|c|}
\hline City & IV & V & VI & VII & VIII \\
\hline Chojnice & $17.0 \%$ & $18.1 \%$ & $17.2 \%$ & $19.5 \%$ & $8.9 \%$ \\
\hline Kalisz & $24.3 \%$ & $27.5 \%$ & $16.7 \%$ & $32.3 \%$ & $18.6 \%$ \\
\hline Kielce & $12.2 \%$ & $12.6 \%$ & $4.6 \%$ & $11.9 \%$ & $11.4 \%$ \\
\hline Leszno & $22.3 \%$ & $23.4 \%$ & $14.3 \%$ & $19.4 \%$ & $20.4 \%$ \\
\hline Łódź & $18.6 \%$ & $22.6 \%$ & $10.9 \%$ & $39.9 \%$ & $10.8 \%$ \\
\hline Poznań & $29.1 \%$ & $21.0 \%$ & $13.1 \%$ & $28.7 \%$ & $28.7 \%$ \\
\hline Toruń & $28.7 \%$ & $26.8 \%$ & $12.0 \%$ & $26.5 \%$ & $10.6 \%$ \\
\hline Warszawa & $25.6 \%$ & $19.2 \%$ & $17.5 \%$ & $32.4 \%$ & $14.4 \%$ \\
\hline
\end{tabular}

Table 13. Extremely dry conditions ( $\mathrm{HTC} \leq 0.4)$, model GFDL, RCP 60, 2060.

\begin{tabular}{|c|c|c|c|c|c|}
\hline City & IV & V & VI & VII & VIII \\
\hline Chojnice & $2.7 \%$ & $2.2 \%$ & $2.0 \%$ & $2.5 \%$ & $1.4 \%$ \\
\hline Kalisz & $7.1 \%$ & $4.1 \%$ & $2.6 \%$ & $9.5 \%$ & $4.8 \%$ \\
\hline Kielce & $1.8 \%$ & $0.9 \%$ & $0.5 \%$ & $1.4 \%$ & $2.5 \%$ \\
\hline Leszno & $5.1 \%$ & $3.4 \%$ & $1.6 \%$ & $4.3 \%$ & $6.0 \%$ \\
\hline Łódź & $4.5 \%$ & $3.1 \%$ & $1.8 \%$ & $11.4 \%$ & $3.2 \%$ \\
\hline Poznań & $7.0 \%$ & $2.9 \%$ & $0.9 \%$ & $7.3 \%$ & $4.9 \%$ \\
\hline Toruń & $7.4 \%$ & $5.4 \%$ & $1.7 \%$ & $4.5 \%$ & $2.8 \%$ \\
\hline Warszawa & $5.1 \%$ & $2.2 \%$ & $3.3 \%$ & $3.3 \%$ & $3.5 \%$ \\
\hline
\end{tabular}


Table 14. Extremely dry or very dry conditions $(\mathrm{HTC} \leq 0.7)$, model GFDL, RCP 60, 2060.

\begin{tabular}{|c|c|c|c|c|c|}
\hline City & IV & V & VI & VII & VIII \\
\hline Chojnice & $13.1 \%$ & $14.4 \%$ & $12.4 \%$ & $14.3 \%$ & $11.3 \%$ \\
\hline Kalisz & $26.3 \%$ & $22.0 \%$ & $14.4 \%$ & $33.7 \%$ & $23.7 \%$ \\
\hline Kielce & $10.1 \%$ & $8.1 \%$ & $4.5 \%$ & $10.3 \%$ & $15.3 \%$ \\
\hline Leszno & $20.9 \%$ & $20.4 \%$ & $11.3 \%$ & $19.8 \%$ & $28.2 \%$ \\
\hline Łódź & $18.4 \%$ & $17.5 \%$ & $10.8 \%$ & $38.1 \%$ & $18.9 \%$ \\
\hline Poznań & $26.6 \%$ & $17.3 \%$ & $7.6 \%$ & $30.1 \%$ & $23.2 \%$ \\
\hline Toruń & $27.0 \%$ & $25.0 \%$ & $10.2 \%$ & $20.6 \%$ & $15.6 \%$ \\
\hline Warszawa & $22.0 \%$ & $14.7 \%$ & $15.6 \%$ & $15.6 \%$ & $20.2 \%$ \\
\hline
\end{tabular}

Table 15. Extremely dry conditions $(\mathrm{HTC} \leq 0.4)$, model HAD, RCP 60, 2060.

\begin{tabular}{|c|c|c|c|c|c|}
\hline City & IV & V & VI & VII & VIII \\
\hline Chojnice & $14.7 \%$ & $7.8 \%$ & $7.0 \%$ & $5.6 \%$ & $5.9 \%$ \\
\hline Kalisz & $16.5 \%$ & $8.7 \%$ & $4.7 \%$ & $13.3 \%$ & $16.1 \%$ \\
\hline Kielce & $9.9 \%$ & $3.4 \%$ & $1.2 \%$ & $2.6 \%$ & $10.4 \%$ \\
\hline Leszno & $10.4 \%$ & $6.8 \%$ & $4.1 \%$ & $8.6 \%$ & $16.6 \%$ \\
\hline Łódź & $17.0 \%$ & $9.5 \%$ & $3.8 \%$ & $17.3 \%$ & $12.1 \%$ \\
\hline Poznań & $17.7 \%$ & $6.6 \%$ & $4.1 \%$ & $10.0 \%$ & $14.6 \%$ \\
\hline Toruń & $25.2 \%$ & $12.9 \%$ & $5.2 \%$ & $6.9 \%$ & $8.6 \%$ \\
\hline Warszawa & $22.0 \%$ & $7.8 \%$ & $7.3 \%$ & $12.3 \%$ & $12.3 \%$ \\
\hline
\end{tabular}

Table 16. Extremely dry or very dry conditions $(\mathrm{HTC} \leq 0.7)$, HAD model, RCP 60, 2060.

\begin{tabular}{|c|c|c|c|c|c|}
\hline City & IV & V & VI & VII & VIII \\
\hline Chojnice & $49.2 \%$ & $43.1 \%$ & $32.5 \%$ & $26.7 \%$ & $28.5 \%$ \\
\hline Kalisz & $50.1 \%$ & $34.8 \%$ & $23.6 \%$ & $44.8 \%$ & $47.1 \%$ \\
\hline Kielce & $38.9 \%$ & $26.8 \%$ & $11.3 \%$ & $18.3 \%$ & $35.7 \%$ \\
\hline Leszno & $35.7 \%$ & $32.0 \%$ & $20.9 \%$ & $32.3 \%$ & $51.6 \%$ \\
\hline Łódź & $52.2 \%$ & $46.2 \%$ & $22.7 \%$ & $51.5 \%$ & $40.5 \%$ \\
\hline Poznań & $52.2 \%$ & $28.9 \%$ & $19.9 \%$ & $41.2 \%$ & $45.5 \%$ \\
\hline Toruń & $67.4 \%$ & $54.3 \%$ & $26.0 \%$ & $33.2 \%$ & $32.2 \%$ \\
\hline Warszawa & $61.4 \%$ & $39.2 \%$ & $30.6 \%$ & $42.8 \%$ & $41.5 \%$ \\
\hline
\end{tabular}

All these results relate to current data that are showned in Tables 17, 18.

Table 17. Extremely dry conditions $(\mathrm{HTC} \leq 0.4)$, current data.

\begin{tabular}{|c|c|c|c|c|c|}
\hline City & IV & V & VI & VII & VIII \\
\hline Chojnice & $2.1 \%$ & $2.1 \%$ & $2.1 \%$ & $2.1 \%$ & $2.1 \%$ \\
\hline Kalisz & $5.2 \%$ & $4.5 \%$ & $2.4 \%$ & $8.7 \%$ & $5.8 \%$ \\
\hline Kielce & $1.6 \%$ & $1.2 \%$ & $0.3 \%$ & $2.0 \%$ & $3.3 \%$ \\
\hline Leszno & $4.3 \%$ & $4.1 \%$ & $1.9 \%$ & $4.6 \%$ & $5.3 \%$ \\
\hline Łódź & $4.3 \%$ & $2.1 \%$ & $1.5 \%$ & $10.5 \%$ & $3.0 \%$ \\
\hline Poznań & $8.6 \%$ & $3.8 \%$ & $1.4 \%$ & $1.4 \%$ & $3.1 \%$ \\
\hline Toruń & $6.6 \%$ & $3.0 \%$ & $1.8 \%$ & $6.4 \%$ & $3.1 \%$ \\
\hline Warszawa & $5.3 \%$ & $2.4 \%$ & $2.0 \%$ & $9.3 \%$ & $4.8 \%$ \\
\hline
\end{tabular}

Table 18. Extremely dry or very dry condition (HTC $\leq$ $0.7)$, current data.

\begin{tabular}{|c|c|c|c|c|c|}
\hline City & IV & V & VI & VII & VIII \\
\hline Chojnice & $12.7 \%$ & $12.7 \%$ & $12.7 \%$ & $12.7 \%$ & $12.7 \%$ \\
\hline Kalisz & $25.1 \%$ & $22.6 \%$ & $14.4 \%$ & $32.6 \%$ & $24.0 \%$ \\
\hline Kielce & $9.5 \%$ & $9.4 \%$ & $3.8 \%$ & $12.0 \%$ & $15.5 \%$ \\
\hline Leszno & $20.1 \%$ & $21.0 \%$ & $12.7 \%$ & $20.1 \%$ & $23.9 \%$ \\
\hline Łódź & $18.1 \%$ & $14.9 \%$ & $10.2 \%$ & $37.2 \%$ & $16.1 \%$ \\
\hline Poznań & $28.9 \%$ & $18.7 \%$ & $10.4 \%$ & $10.4 \%$ & $18.3 \%$ \\
\hline Toruń & $25.7 \%$ & $20.8 \%$ & $10.9 \%$ & $25.0 \%$ & $15.1 \%$ \\
\hline Warszawa & $23.1 \%$ & $15.3 \%$ & $13.2 \%$ & $34.0 \%$ & $20.9 \%$ \\
\hline
\end{tabular}

These probabilities were calculated based on the probability density of Gamma distributions. This distribution was chosen because it best approximated HTC values in the vegetation periods in Poland. The calculations were made using the fitdistrplus function in $\mathrm{R}$ software [25]. Below is a comparison of all screenplays for June (Fig. 2 - 9).

Designations used in figures $2-9$ :

(1) - current data,

(2) - GFDL RCP4.5 2040,

(3) - GFDL RCP4.5 2060,

(4) - GFDL RCP6.0 2040,

(5) - GFDL RCP6.0 2060,

(6) - HAD RCP4.5 2040,

(7) - HAD RCP4.5 2060,

(8) - HAD RCP6.0 2040,

(9) - HAD RCP6.0 2060.

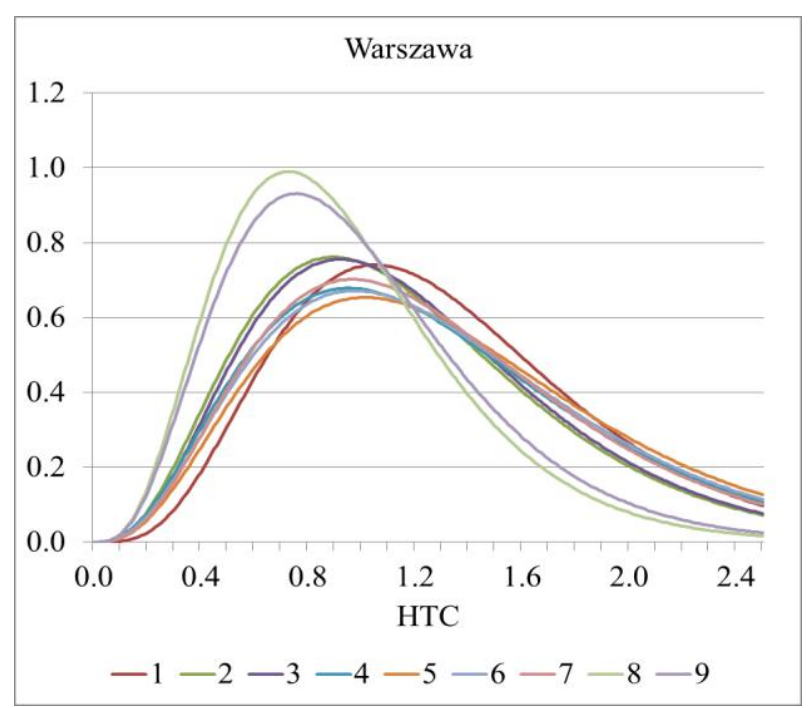

Fig. 2. Warszawa - June - estimated probability density function for various weather scenarios. 


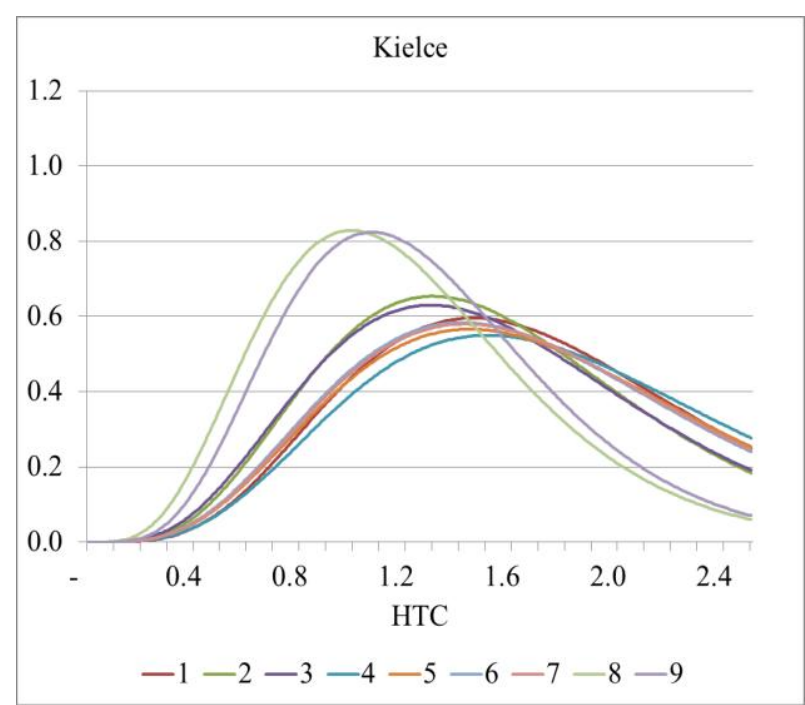

Fig. 3. Kielce - June - estimated probability density function for various weather scenarios.

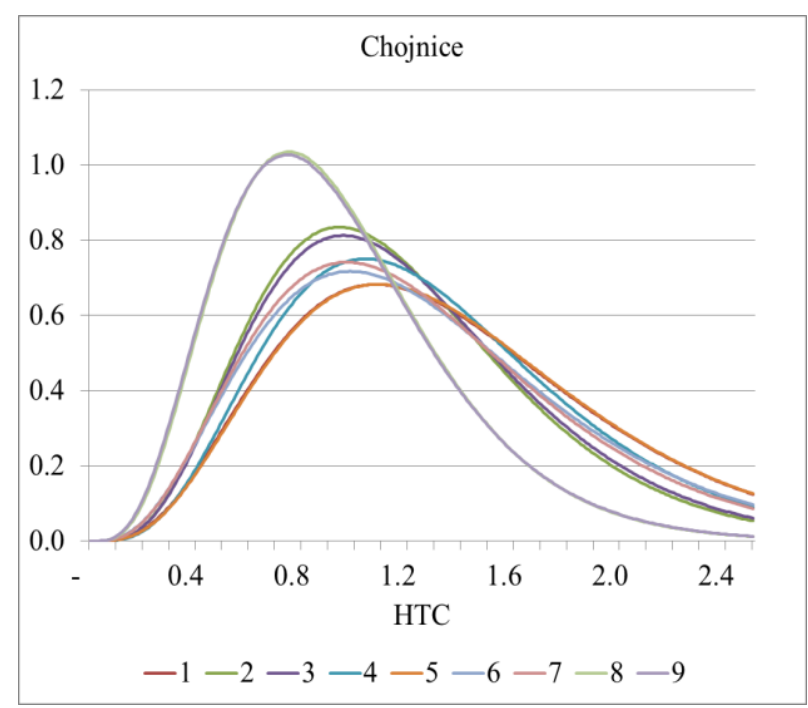

Fig. 4. Chojnice - June - estimated probability density function for various weather scenarios.

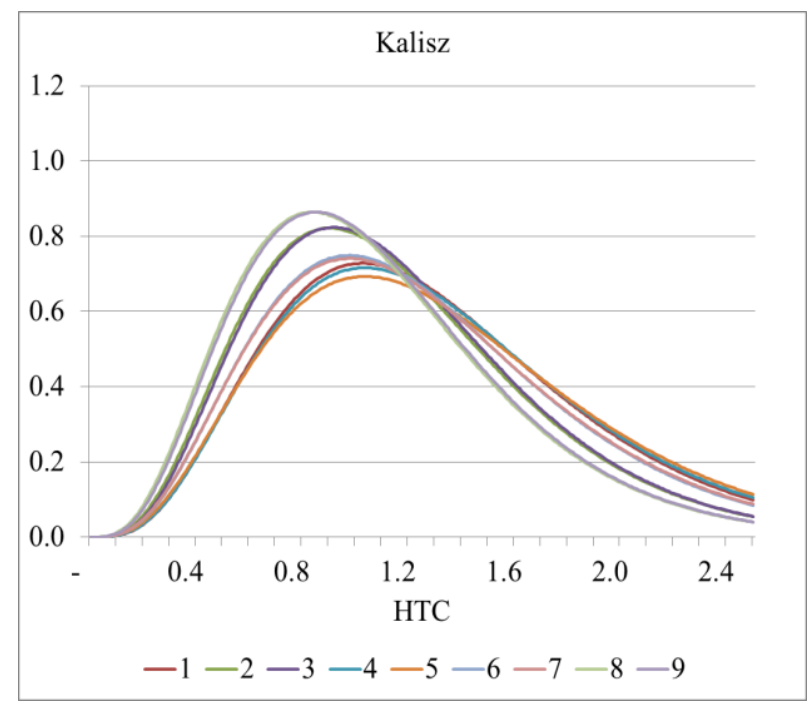

Fig. 5. Kalisz - June - estimated probability density function for various weather scenarios.

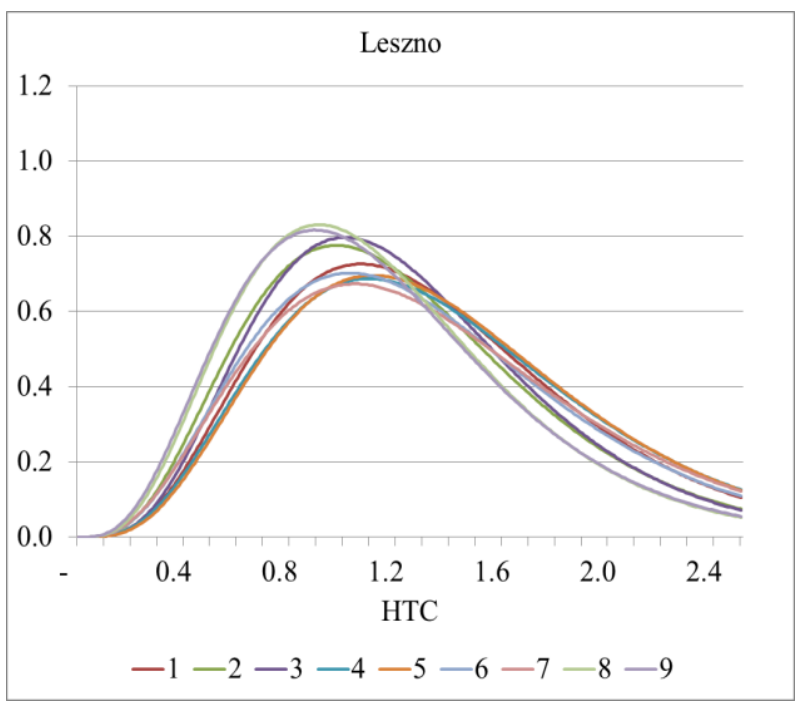

Fig. 6. Leszno - June - estimated probability density function for various weather scenarios.

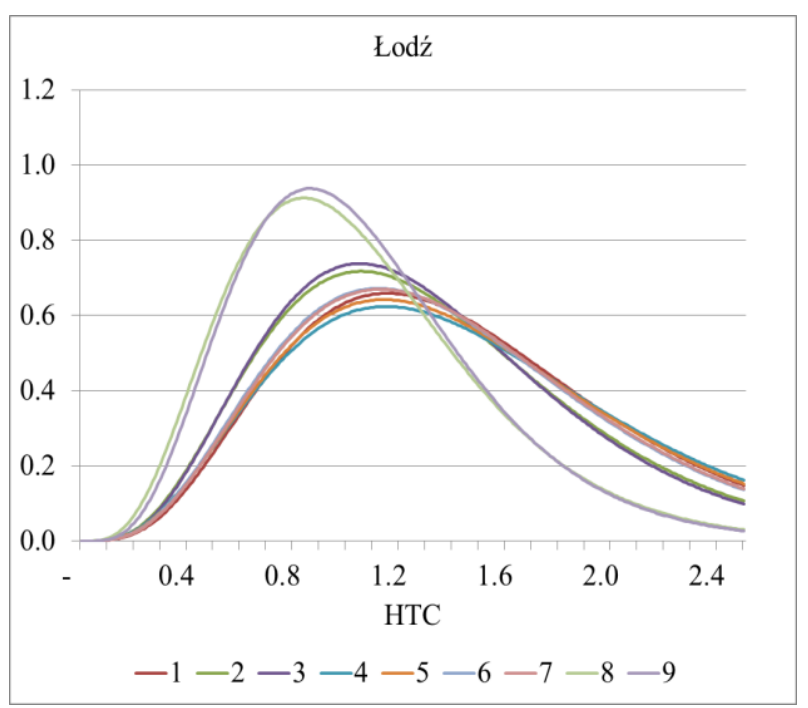

Fig. 7. Łódź - June - estimated probability density function for various weather scenarios.

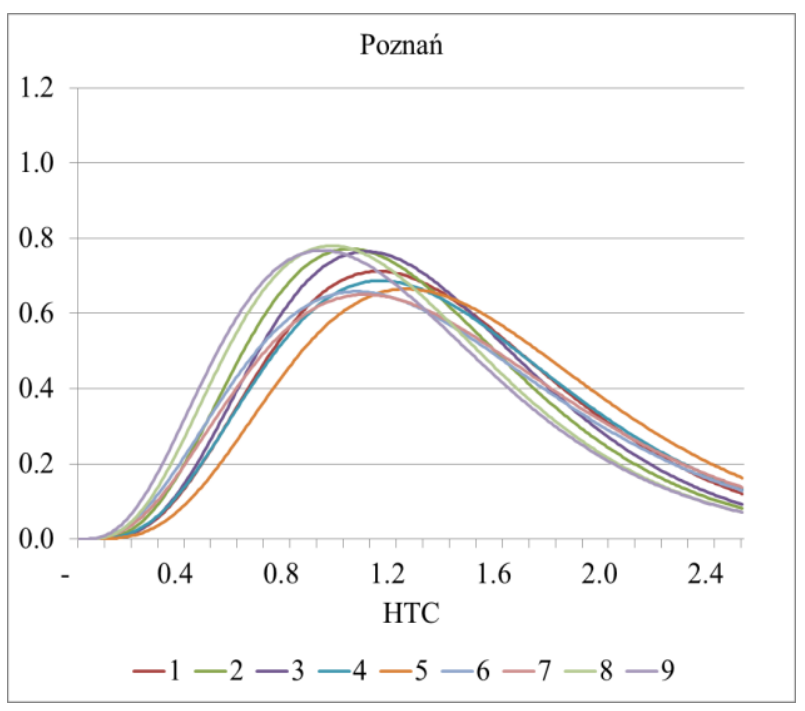

Fig. 8. Poznań - June - estimated probability density function for various weather scenarios. 


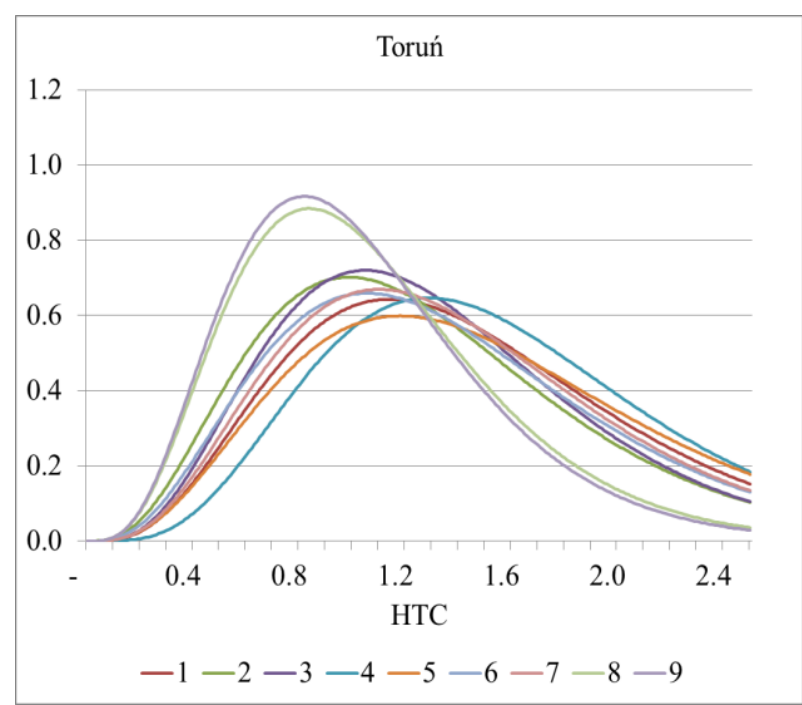

Fig. 9. Torun - June - estimated probability density function for various weather scenarios.

\section{Conslusions}

The lowest probability of the occurrence of extremely dry and very dry states occurs most frequently in Kielce. For the fixed model, the highest values occur most often for July. For the same model, no increase was observed due to extension of the time horizon. For the same time horizon and another RCP, there was no increase in the value of HTC due to higher RCP. Some of the highest probability of drought occurs in central part of Poland.

\section{References}

1. L. Łabędzki, Susze rolnicze. Zarys problematyki oraz metody monitorowania i klasyfikacji (IMUZ, Falenty, 2006)

2. M. Miętus, Ekstremalne zjawiska hydrologiczne i meteorologiczne (PTGF IMGW, Warszawa, 2005)

3. J. Żarski, Infrastructure and Ecology of Rural Areas 5, 29-37 (2011)

4. A. Ziernicka-Wojtaszek, Water-Environment-Rural Areas 12(2), 365-376 (2012)

5. W. Treder, Infrastructure and Ecology of Rural Areas 5, 29-37 (2011)

6. L. Kuchar, S. Iwański, E. Gąsiorek, E. Diakowska, Infrastructure and Ecology of Rural Areas 2(1), 319 -334 (2015)

7. L. Kuchar, S. Iwański, E. Gąsiorek, E. Diakowska, Infrastructure and Ecology of Rural Areas 1(2), 257 -273 (2017)

8. L. Kuchar, S. Iwański, Infrastructure and Ecology of Rural Areas 2, 187-200 (2013)

9. A. Doroszewski, J. Jadczyszyn, J. Kozyra, R. Pudełko, T. Stuczyński, K. Mizak, A. Łopatka, P. Koza, T. Górski, E. Wróblewska, WaterEnvironment-Rural Areas 12(2), 77-91, (2012)
10. S. Dudek, R. Kuśmierek-Tomaszewska, J. Żarski, Infrastructure and Ecology of Rural Areas 3, 109117 (2009)

11. IPCC Fourth Assessment Report: Climate Change, 2007 (AR4)

12. IPCC Fifth Assessment Report: Climate Change, 2013 (AR5)

13. L. Kuchar, S. Iwański, Infrastructure and Ecology of Rural Areas 5, 7-18 (2011)

14. G.A. Schmidt, R. Ruedy, J.E. Hansen, I. Aleinov, N. Bell, M. Bauer, S. Bauer, B. Cairns, V. Canuto, Y. Cheng, A. Del Genio, G. Faluvegi, A.D. Friend, T.M. Hall, Y. Hu, M. Kelley, N.Y. Kiang, D. Koch, A.A. Lacis, J. Lerner, K.K. Lo, R.L. Miller, L. Nazarenko, V. Oinas, J. Perlwitz, J. Perlwitz, D. Rind, A. Romanou, G.L. Russell, M. Sato, D.T. Shindell, P.H. Stone, S. Sun, N. Tausnev, D. Thresher, M. Yao, J. Climate 19, 153-192 (2006)

15. V. K. Boken, A. P. Cracknell, R. L. Heathcote, Monitoring and Predicting Agricultural Drought: A Global Study (Oxford University Press, New York, 2005)

16. L. Kuchar, S. Bac, Aktualne problemy rolnictwa, gospodarki żywnościowej $i$ ochrony środowiska (AR, Wrocław, 2006)

17. B. Skowera, J. Puła, Acta Agrophys. 3(1), 171-177 (2004)

18. G.T. Sieljaninov, Agroclimatic Map of the World (Gidrometizdat, Leningrad, 1966)

19. G. Evarte-Bundere, P. Evarts-Bunders, Acta Biol. Univ. Daugavp. 12(2), 135-148 (2012)

20. M. Monteva, V. Kazandjiev, V. Georgijeva, IWTC 14, Cairo, Egypt, 3-14 (2010)

21. L. Kuchar, Mathematics and Computer in Simulation 65, 69-75 (2004)

22. L. Kuchar, S. Iwanski, 10th Conference on Interdisciplinary Problems in Environmental Protection and Engineering EKO-DOK 44 (2018)

23. L. Kuchar, Water-Environment-Rural Areas 5, 185 (2005)

24. G. A. Baigorria, J. W. Jones, J. Clim 23, 5990 (2010)

25. M. L. Delignette-Muller, C. Dutang, Journal of Statistical Software 64(4) (2015) 